\title{
Quantitative Investigation of Aggregate Skeleton Force Chains of Asphalt Mixtures Based on Computational Granular Mechanics
}

\author{
Guoqiang Liu, Dongdong Han, and Yongli Zhao (i) \\ School of Transportation, Southeast University, Nanjing, Jiangsu 210096, China \\ Correspondence should be addressed to Yongli Zhao; yonglizhao2016@126.com
}

Received 22 December 2019; Accepted 24 April 2020; Published 11 May 2020

Academic Editor: Yuqing Zhang

Copyright $(2020$ Guoqiang Liu et al. This is an open access article distributed under the Creative Commons Attribution License, which permits unrestricted use, distribution, and reproduction in any medium, provided the original work is properly cited.

\begin{abstract}
For asphalt mixtures, the difference between strong force chains (SCF) can reflect the skeleton performance. In this paper, six kinds of mineral mixture discrete element model were established. And various SCF evaluation indices of different mineral mixtures were calculated. Results indicate that the short length SCF number proportions of dense-skeleton type mineral mixtures are higher than that of dense-suspended type mineral mixtures under the same nominal maximum aggregate size (NMAS). And the NMAS has a great influence on the SCF length cumulative proportions, and different NMAS can significantly change the stress transfer path for dense-suspended type mixture. Nevertheless, the SCF length cumulative proportions have consistency for denseskeleton type mixtures. The small SCF alignment coefficient proportions of dense-suspended type mixtures are higher than that of dense-skeleton type mixtures. In particular, under larger NMAS, the difference is more obvious. The SCF that is close to straight line is conducive to transfer loading. Therefore, dense-skeleton type mixture has better rutting resistance. The SCF bears the main loading for mixtures. Mixtures stone matrix asphalt (SMA) has a stronger bearing capacity than that of mixtures AC under the same NMAS. These findings provide insight into the mechanics of skeleton structure.
\end{abstract}

\section{Introduction}

Rutting is one of main forms of asphalt pavement diseases, and its cause is that the asphalt mixture produces too much cumulative deformation under the action of high temperature or the loading for a long time [1]. According to the SHRP program, the skeleton structure formed by mineral aggregates is the main carriers to resist and transfer external loading, and the mineral aggregate skeleton structure contributes $70 \%$ to the high temperature stability of asphalt mixture [2]. Therefore, it is necessary to study the skeleton structure of asphalt mixture.

With an increase of digital image processing (DIP) technology application in the field of road engineering, the research on skeleton structure has been studied deeply from macroscale to mesoscale and gradually focuses on the contact characteristics of coarse aggregates. Coenen et al. [3] and Cai et al. [4] determined the contact distance threshold based on the minimum calculated aggregate size. Sefidmazgi et al. [5-7] defined the contact length $L$ and contact orientation $\Delta \theta$ to characterize the aggregate contact properties. Shi et al. [8, 9] and Ying et al. [10] also proposed some mesoindices and attempted to establish the relationship between aggregate contact properties and gradations. Jiang et al. $[11,12]$ defined the indices from the contact distance, contact length, and contact orientation and analyzed the relation between skeleton microstructure and marcoperformance, especially the antirutting performance. The above research shows that the geometric composition of skeleton structure has been studied deeply. However, the skeleton structure is the main body of asphalt mixture to resist and transfer external loading. And it is difficult to reflect the essential characteristics of skeleton structure mechanical behavior only characterizing its geometric composition.

Other researchers have recognized the shortcomings of the studies mentioned above and attempt to analyze the mechanical behavior of asphalt mixture skeleton structure. Chen et al. [13] utilized discrete element method (DEM) to evaluate aggregate structure characteristics and their 
resistance to deformation. The results indicate that the quality evaluation of asphalt mixture skeleton structure depends on the stress magnitude transferred by the skeleton structure and its ability to resist external loading. Ma et al. [14] analyzed the micromechanical response of skeleton structure during the virtual wheel tracking tests by DEM. The results show that the aggregate movement reduces the skeleton structure stability, which is mainly represented by the decrease of effective contact number and increase of contact force in the skeleton structure. Li et al. [15] developed aggregate slip test to evaluate aggregate slip behavior in the skeleton structure. And the slip energy index (SEI) is the best parameter to evaluate the stability of asphalt mixture skeleton structure. Wang et al. [16] analyzed the skeleton strength of porous asphalt mixtures by a penetration test. It is found that the percent passing of $9.5 \mathrm{~mm}$ has the largest impact on the skeleton strength. Zhang et al. [17] evaluated the contribution rate of various mineral aggregate to make the skeleton structure stabilization and transfer external loading in asphalt mixture. The results show that based on the contribution rate difference, the mineral aggregate with different sieve size can be distinguished as main bearing structure, main stable structure, bearing structure, and stability structure.

According to the granular mechanics theory, the contact between adjacent granules forms many paths for transferring external loading, which are called force chains. The force chains interweave to form force chains network through the granular material system, which determines the macroscopic mechanical properties of granular material system [18]. At present, researchers in the field of pavement materials have begun to pay attention to the force chains structure of skeleton structure. Chen and Li. [19] established the crumb rubber asphalt mixture discrete element models and qualitatively analyzed the force chains of crumb rubber asphalt mixtures. The results indicate that the SFC are mainly composed of large size coarse aggregates; the weak force chains are composed of fine aggregates. Shi et al. [20] compared the force chains structure of SMA13 and AC13 and found that the bearing capacity of SMA13 mixture was at least four times than that of $\mathrm{AC} 13$. The results indicates that whether the contact point is located on SFC should be the only criterion to determine the effectiveness of aggregate contact. Chang et al. [21] defined the force chains angle index and quantitatively analyzed the evolution and distribution of force chains between aggregates in asphalt mixture. The results show that it is feasible to characterize the force chains, and the characteristics of force chains can reflect the internal load transfer law of asphalt mixture.

The above studies show that the skeleton structure has always been one of the research focuses in the field of pavement materials. Moreover, the skeleton structure has been investigated deeply from the geometry composition to the mechanical behavior. However, the existing research on mechanical behavior of skeleton structure is more about the statistical analysis of contact force, which is difficult to reflect the essential characteristics of bearing and loading and transitive behavior of skeleton structures. In asphalt mixture, force chains are an effective tool to reflect the ability of transfer external loading. Nevertheless, the existing research studies about asphalt mixture force chains are mainly qualitative analysis, lacking quantitative investigation. Therefore, this paper attempts to quantify the force chains and establish the relationship between the ability of transfer external loading and skeleton structure.

\section{Objective and Scope}

The objectives of this study are to quantitatively compare the force chains characteristics of mineral aggregate skeleton structure. In order to achieve this goal, the two main elements are the following:

(1) To analyze the existing force chains identification criteria and develop force chains identification algorithm for differentiating the strong and weak force chains of different mineral mixtures

(2) To propose the force chains performance indices for characterizing the properties of skeleton structure force chains and compare the geometrical characteristics and bearing capacity of different skeleton mixture force chains

\section{Virtual Specimen Generation and Micromechanical Parameters}

3.1. Virtual Specimen Generation. As we all know, the volume ratio of mineral mixture is more than $85 \%$ in asphalt mixture [22], and so the mechanical behavior of mineral mixture can reflect the mechanical performance of asphalt mixture to a certain extent. Thus, the mineral mixture was selected as research object in this study. And the gradation median of asphalt mixtures AC13, AC16, AC20, SMA13, SMA16, and SMA20 was selected to fabricate virtual specimens according to the Chinese specification, JTG F40-2004 [23]. The gradations of mineral mixtures were shown in Figure 1, respectively. In order to simplify analysis, the effect of aggregate morphology on the force chains properties was not considered in this paper. Therefore, aggregates were assumed to be round granules. The aggregates of which diameters were equal or greater than $2.36 \mathrm{~mm}$ were selected, and the aggregate total number was set to 1000 to improve the model computational efficiency. The DEM model was established by two-dimensional Particle Flow Code (PFC2D) software to conduct virtual penetration test, and the modeling processing was divided into three cycles, including granular generation followed by the corresponding compaction, as shown in Figure 2. Six virtual specimens for uniaxial penetration test were displayed in Figure 3, respectively.

Prior to simulation, a wall with length of $50 \mathrm{~mm}$ was generated above the container. The wall moved downward with $1.27 \mathrm{~mm} / \mathrm{min}$ to penetrate mineral mixtures. The wall 


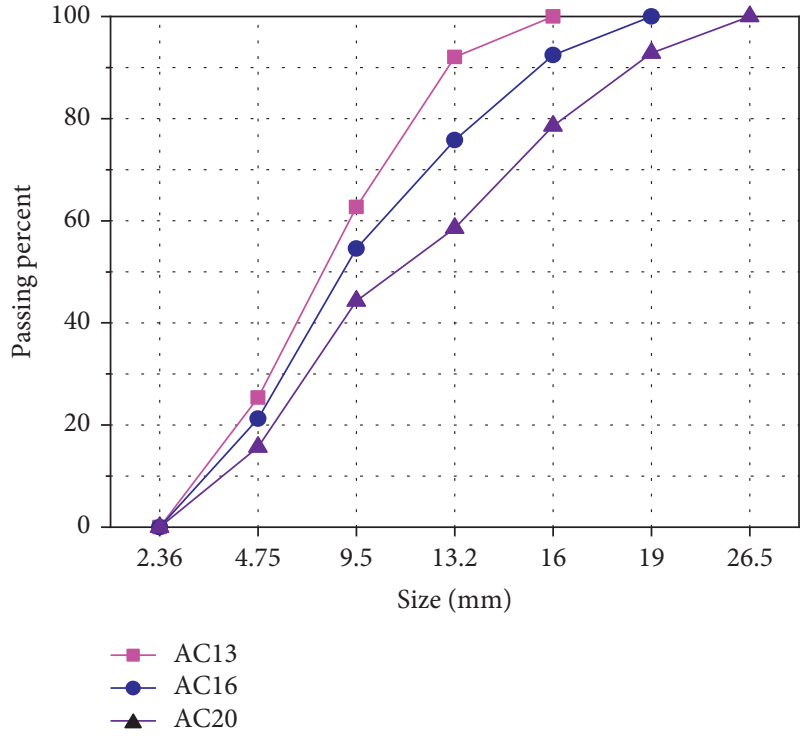

(a)

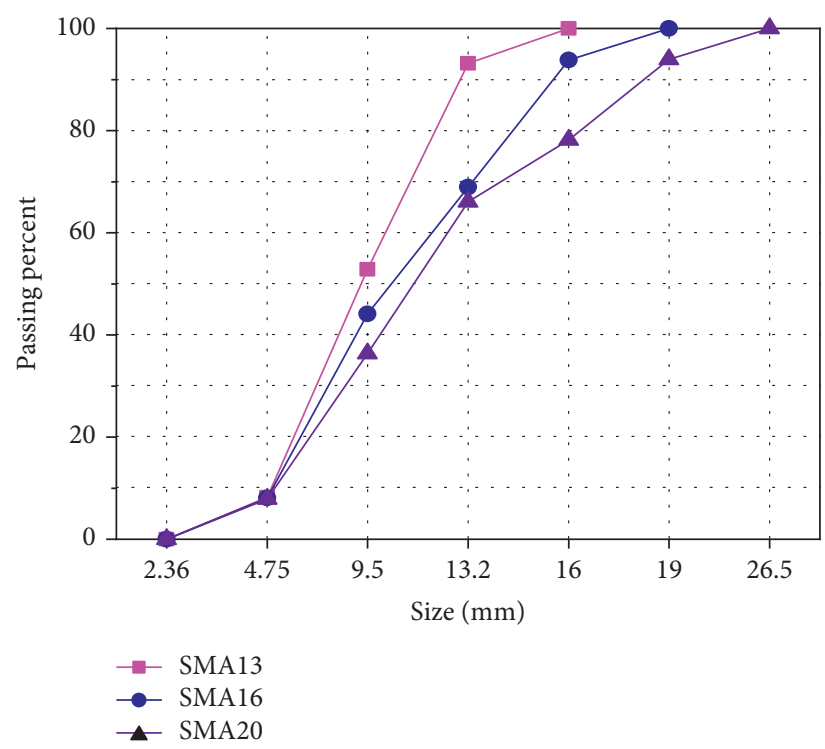

(b)

Figure 1: Gradation of different mineral mixtures. (a) Dense-suspended type; (b) dense-skeleton type.

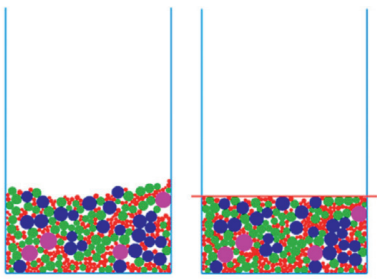

First cycle

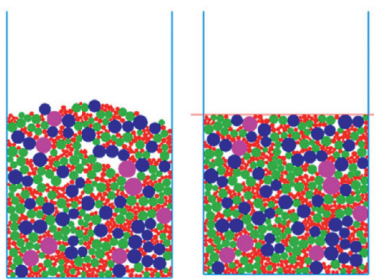

Second cycle

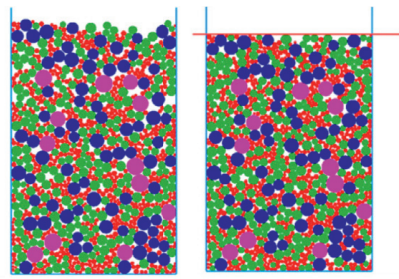

Third cycle

Figure 2: Compaction process in three cycles.

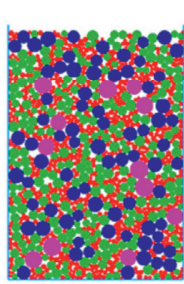

$\mathrm{AC} 13$

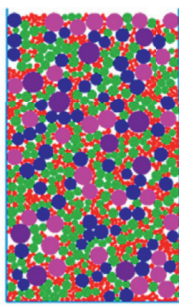

AC16

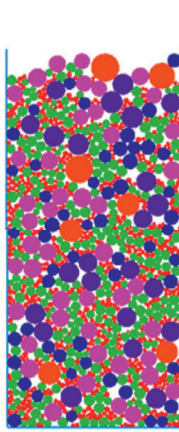

AC20

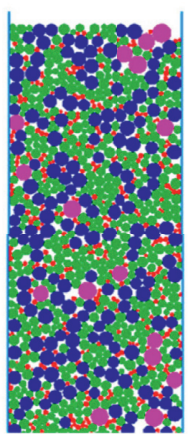

SMA13

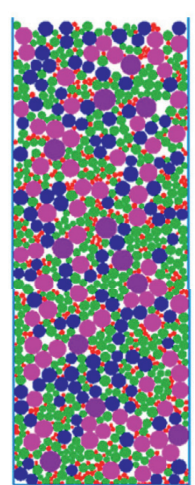

SMA16

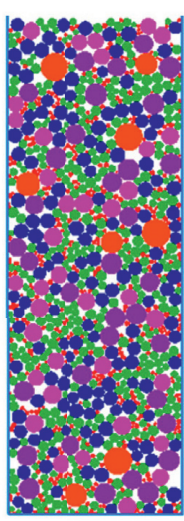

SMA20

Figure 3: Virtual specimen of different mineral mixtures.

moves down until the penetration depth reaches $10 \mathrm{~mm}$ to stop penetration test.

3.2. Micromechanical Parameters for DEM Models. Based on previous studies $[24,25]$, the coarse aggregates were regarded as pure elastic materials and two micromechanical models including stiffness model and slip model were used during the DEM simulation. The stiffness model characterizes the relationship between the normal/ shear contact force and the relative normal/shear displacement of the contacted entities. The contact force is determined by 


$$
\begin{gathered}
K^{n}=\frac{k_{n}^{A} k_{n}^{B}}{k_{n}^{A}+k_{n}^{B}}, \\
k^{s}=\frac{k_{s}^{A} k_{s}^{B}}{k_{s}^{A}+k_{s}^{B}}, \\
F_{i}^{n}=K^{n} U^{n} n_{i}, \\
\Delta F_{i}^{s}=-k^{s} \Delta U_{s},
\end{gathered}
$$

where $K^{n}$ is the normal contact stiffness; $k_{n}^{A}$ and $k_{n}^{B}$ are normal stiffness of entities $\mathrm{A}$ and $\mathrm{B}$, respectively. $k^{s}$ is the shear contact stiffness; $k_{s}^{A}$ and $k_{s}^{B}$ are shear stiffness of entity $\mathrm{A}$ and entity B, respectively. $F_{i}^{n}$ is the normal contact force between two entities, which is a secant stiffness because it relates the total normal force to the total normal displacement; $U^{n}$ is the overlap of the two contact entities along the normal direction; $\Delta F_{i}^{s}$ is the shear contact force between two entities, which is a tangent stiffness because it relates the increment of shear force to the increment of the shear displacement; and $i$ is the direction of contact.

The slip model is an intrinsic property of two entities (ball-ball/ball-wall) in contact. It provides no normal strength in tension and allows the slip to occur by limiting the shear force. Contact is checked for slippery conditions by calculating the maximum allowable shear contact force in (5). The slip will occur until the shear force exceeds the maximum allowable value. One has

$$
F_{\max }^{s}=\mu\left|F_{i}^{n}\right|
$$

where $F_{\max }^{s}$ is the maximum allowable shear contact force; $F_{i}^{n}$ is the normal contact force between two entities; and $\mu$ if the friction coefficient in PFC2D.

At present, it is often used to obtain the micromechanical parameters through the macroresponses of granular materials. According to previous studies [13, 26, 27], the appropriate micromechanical parameters were determined after many times trial calculation and adjustment. The adjusted micromechanical parameters are shown in Table 1.

\section{Strong and Weak Force Chains Criteria and Identification Algorithm}

4.1. Strong and Weak Force Chains Criteria. In granular materials, the force chain structure is divided into strong and weak force chains according to the magnitude of transfer loading, and the strong chain structure determines the macroscopic mechanical behavior of granular materials [18]. Hence, the identification of strong and weak force chains is the basis of analyzing the force chains of skeleton structure.

Initially, researchers distinguished the strong and weak force chains from the geometric view. Cates et al. [28] believed that the strong force chains (SFC) are a linear chain formed by three or more contiguous granules. Howell et al. [29] conducted low-speed shear tests on two-dimensional granular materials and defined that the SFC was collinear disks. With the understanding of force chain deepening, researchers began to identify SFC in terms of stress. Peters et al. [30] suggested that the SFC should meet the three requirements: (1) SFC should be formed by three or more granules; (2) the absolute value of major principal stress should be more than or equal to the average value of system; (3) the angle between two adjacent normal contacts must be less than or equal to $\alpha$, and $\alpha$ is $45^{\circ}$. Sun and Wang [31] deemed that the coordination number is directly related to the microstructure of the contact network, and so $\alpha$ should be determined by coordination number $\left(\alpha=180^{\circ} /\langle Z\rangle,\langle Z\rangle\right.$ is the average coordination number).

It can be seen from the above literature that the SFC structure should meet the requirements of contact force, quasilinearity, and length. However, there is no unified SFC standard for specific requirements. In this study, the standard proposed by Sun and Wang [31] is referenced. The SFC of mineral mixture skeleton structure should meet the following three conditions:

(1) Contact force threshold: the contact force within the strong force chains needs to be equal or greater than the average contact force

(2) Contact angle threshold: the angle between two adjacent normal contacts must be less than or equal to the threshold value $\theta c . \theta c=180^{\circ}$ divided by the average coordination number

(3) Granular number threshold: the granular number of force chains must be greater than two

4.2. SFC Identification Algorithm. According to the strong and weak force chains criteria, the SFC identification algorithm is developed based on platforms of PFC2D and MATLAB2019a. The specific steps for searching SFC are as follows, and the single SFC identification flowchart is shown in Figure 4. The identified strong and weak force chains of AC13 are seen in Figure 5.

(1) Output aggregate and contact information via PFC2D. The contacts that do not satisfy the contact force threshold value are deleted.

(2) Determine the initial aggregate particle, set as particle A. If $F$ between particle $\mathrm{A}$ and adjacent particle is larger than the average contact force, the adjacent particle is set as particle $B$.

(3) Particle B has many adjacent particles, only particles $\mathrm{C}$ and $\mathrm{D}$ are within the angle threshold. If only the contact force between particle $\mathrm{B}$ and particle $\mathrm{C}$ is greater than the average contact force, then particle $\mathrm{C}$ constitutes the third particle in the force chains.

(4) If more particles which are $F$ are greater than the average contact force within the angle threshold, then the minimum angle of the particle is selected, and repeat the above steps.

\section{Evaluation Indices for SFC of Mineral Mixtures}

5.1. SFC Length. Force chains are the paths of transferring external loading in asphalt mixtures. Therefore, SFC length 
TABLE 1: Micromechanical model parameters.

\begin{tabular}{lccc}
\hline Contact type & Normal contact stiffness, Kn $(\mathrm{N} / \mathrm{m})$ & Shear contact stiffness, ks $(\mathrm{N} / \mathrm{m})$ & Friction coefficient, $\mu$ \\
\hline Ball-ball & $2.0 \mathrm{e} 8$ & $2.0 \mathrm{e} 8$ & 0.5 \\
Ball-facet & $2.0 \mathrm{e} 9$ & $2.0 \mathrm{e} 9$ & 0.5 \\
\hline
\end{tabular}

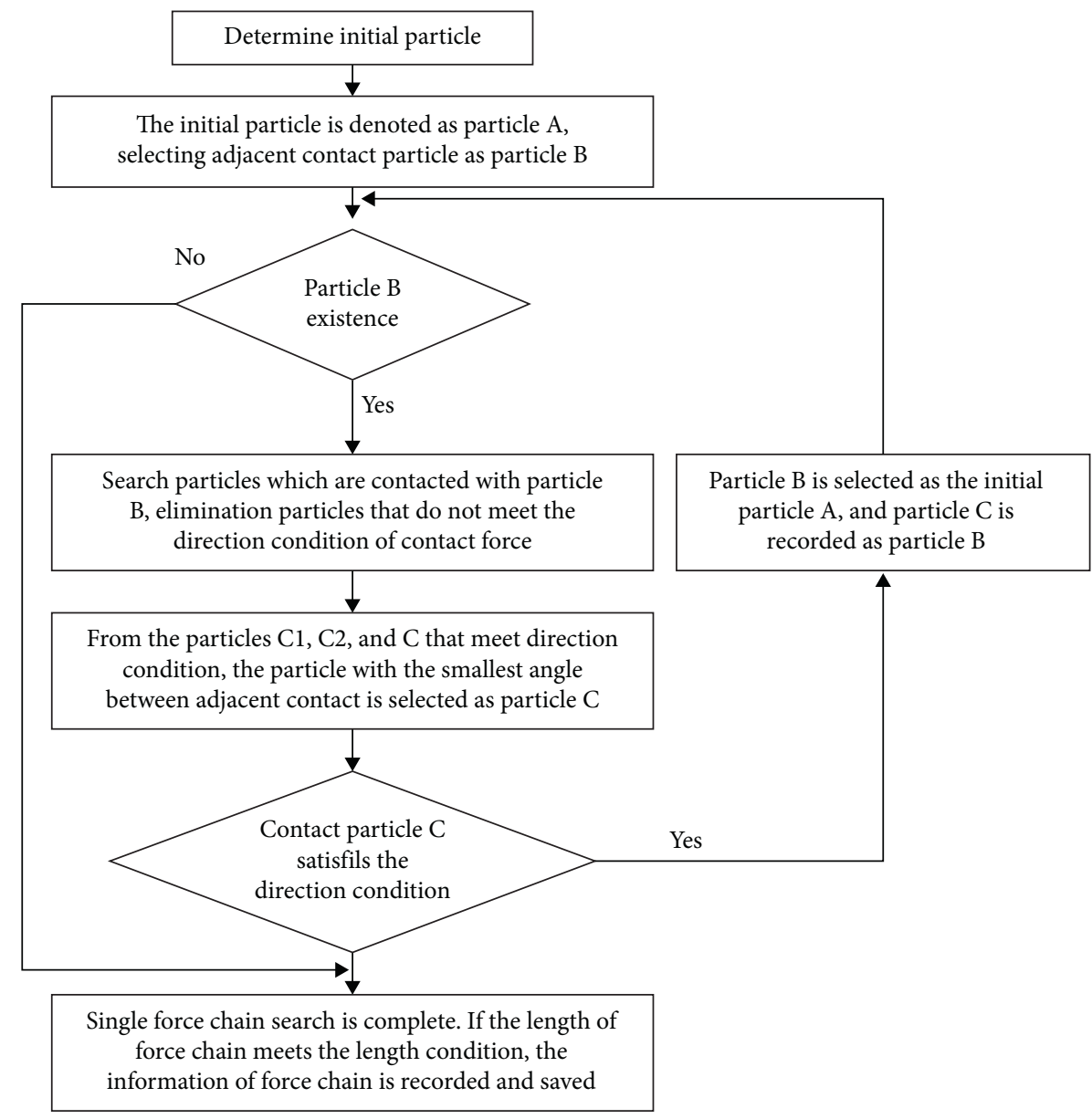

FIGURE 4: Flowchart of single SFC identification.

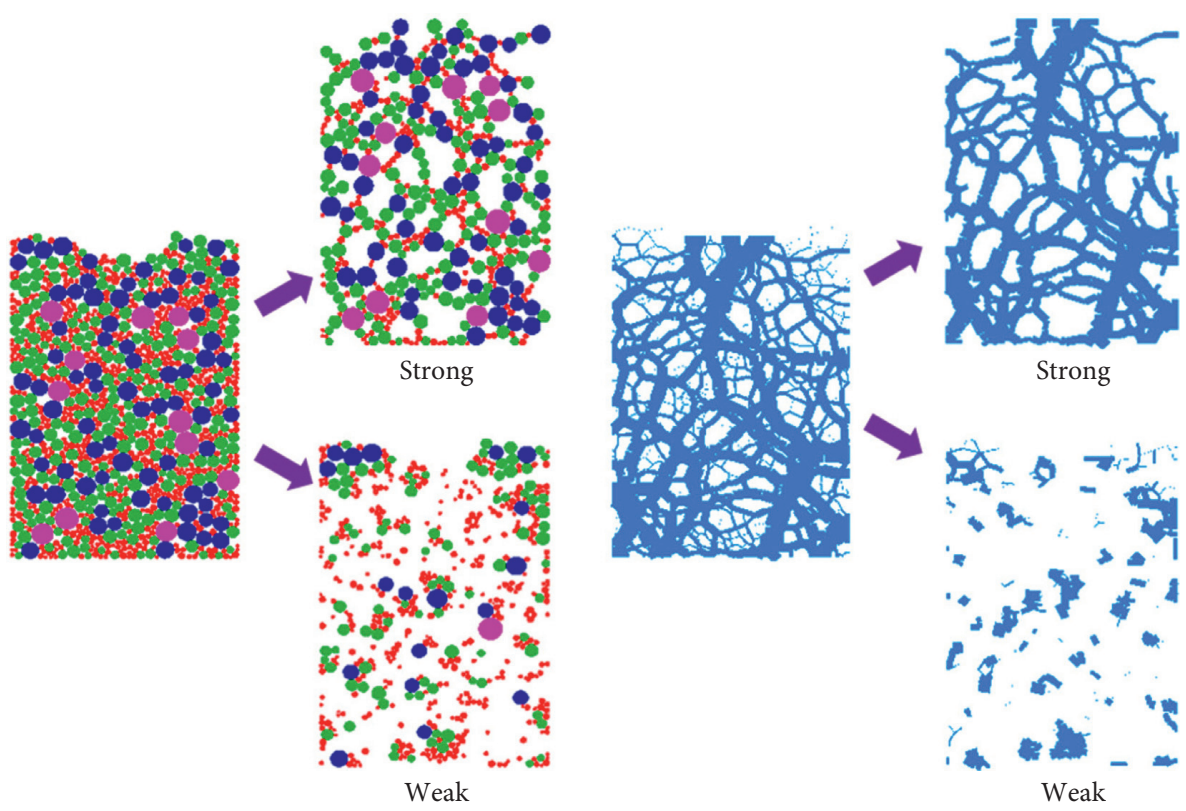

FIgURE 5: Strong and weak force chains of AC13. 
is an important index to reflect the length of path. In this study, the granular number is the same for different DEM models. Thus, the SFC length is normalized by DEM model height. The length of SFC is calculated via

$$
l_{i}=\sum_{j=1}^{N_{i}-1} \frac{d_{j, j+1}}{l_{m}}
$$

where $d_{j, j+1}$ is the distance between particle $j$ and particle $j+1$ mass center; $N_{i}$ represents the particle number of $i$ th SFC; $1 \mathrm{~m}$ is the DEM model height.

5.2. SFC Alignment Coefficient. The SFC linetype is another feature that affects the stability of transfer external loading. Hence, the alignment coefficient is defined to characterize the linetype of SFC. And the SFC alignment coefficient is calculated via

$$
\delta_{i}=1-\frac{\sum_{p=1}^{M} \alpha_{p}}{180^{\circ} \times M},
$$

where $\alpha_{p}$ represents the $p$ th angle between adjacent normal directions of ith SFC. $M$ is the total number of adjacent contacts. The smaller the alignment coefficient of SFC is, it is easier to break. The larger the alignment coefficient of SFC is, the more stable SFC is.

5.3. SFC Bearing Ratio. The bearing capacity difference of different SFC structure is great. The difference can be used to compare the skeleton structure of different asphalt mixtures. In this study, the bearing capacity is defined as the ratio between the sum of normal contact force of SFC structure and the sum of normal contact force of total force chains, as shown in

$$
P_{s}=\frac{\sum_{\text {Strong }}\left(\left|f_{i}\right|\right)}{\sum\left(\left|f_{j}\right|\right)} \times 100 \%,
$$

where $\sum_{\text {Strong }}\left(\left|f_{i}\right|\right)$ represents the sum of normal contact force of SFC; $\sum\left(\left|f_{j}\right|\right)$ represents the sum of normal contact force of total force chains.

\section{Results and Discussions}

6.1. SFC Number of Different Mineral Mixtures. Based on the strong and weak force chains criteria, the SFC structures of different mineral mixtures are identified by the proposed SFC identification algorithm, as shown in Figure 6. And the SFC number is counted, as detailed in Figure 7.

Figure 6 shows that, for dense-suspended mixtures, the change trend of SFC network structure of mixture under different NMAS does not present obviously regularity. However, for dense-skeleton mixtures, mixture SMA13 has the more intricate SFC network structure than that of SMA16 and SMA20. There is a small difference between the SFC network of SMA16 and SMA20.

Data in Figure 7 suggest that, for dense-suspended mineral mixtures, AC13 has more SFC number than that of AC16 and AC20; AC16 has the minimum SFC number. For the dense-skeleton mineral mixtures, the SFC number decreases with an increase in NMAS. The results indicate that, for mineral mixtures, the complexity of SFC structure is high under small NMAS, which is not beneficial to improve the efficiency of transfer loading.

6.2. SFC Length of Different Mineral Mixtures. SFC length can reflect the path length of transferring external loading in asphalt mixtures. In this study, the SFC length of different mineral mixtures is calculated by programming. And the SFC length distribution, minimum length of SFC, maximum length of SFC, and SFC length cumulative distribution of different mineral mixtures are shown in Figures 8 11, respectively.

It can be observed from Figure 8 that, in general, the number proportion of short length SFC is much higher than that of long length SFC for all mineral mixtures which indicate that the stress transfer of mineral mixture follows the shortest path principle. The maximum length SFC is about one. Therefore, the short length SFC is defined as the strong force chains with a length no longer than 0.3. Comparing the SFC length distribution of AC13 and SMA13, the short length SFC number proportions of AC13 and SMA13 are $55.83 \%$ and $67.23 \%$, as shown in Table 2 . The short length SFC number proportion of SMA13 is more than that of AC13. The short length SFC is beneficial to transfer external loading, and so the ability to bear and transfer loading of SMA13 is stronger than AC13. It can be observed from Figure 8(b) and 8(c) that the short length SFC number proportion of AC16 and AC20 is $51.38 \%$ and $64.04 \%$ severally. And the short length SFC number proportion of SMA16 and SMA20 is $65.46 \%$ and $65.74 \%$, respectively. It also illustrates that the short length SFC number proportions of dense skeleton type mixtures are higher than that of dense-suspended type mixtures under the same NMAS. These results further indicate that the short length SFC is more stable than long length SFC and is beneficial to bear and transfer loading. Therefore, the dense skeleton type mixtures have better rutting resistance than that of dense suspended type mixtures.

Figure 9 shows that, on the whole, the minimum length SFC decreases with an increase in NMAS no matter what kind of mixtures. The mixtures AC and SMA have a similar minimum length SFC. The minimum length SFC of AC16 and AC20 is longer than that of SMA16 and SMA20, respectively. Moreover, the difference increases with an increase in NMAS. The results show that the ability of transfer external loading of dense-skeleton type mixtures is stronger than that of dense-suspended type mixtures. It can be seen from Figure 10 that the maximum length SFC of mixtures $\mathrm{AC}$ is longer than that of mixtures SMA under NMAS $16 \mathrm{~mm}$ and $19 \mathrm{~mm}$. Although the maximum length SFC of AC13 is shorter than SMA13, the long length SFC number proportion of AC13 is higher than that of SMA13 according to the Figure 8(a). We infer that the long length SFC is unstable, and short length SFC is conducive to transfer external loading. This is one of mechanics' mechanisms why dense-skeleton type mixture has a better bearing capacity than dense-suspended type mixture. 


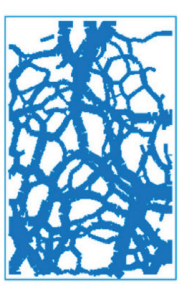

$\mathrm{AC} 13$

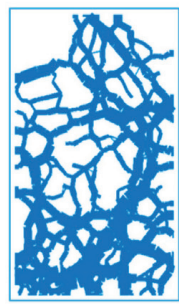

AC16

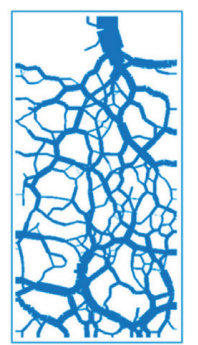

AC20

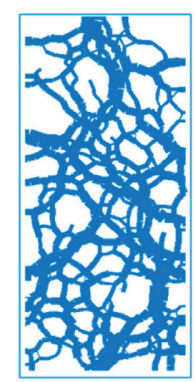

SMA13

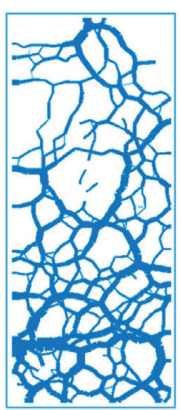

SMA16

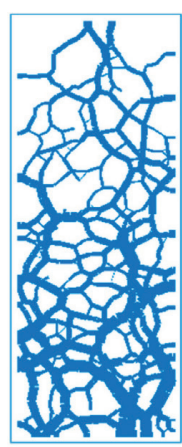

SMA20

FIGURE 6: SFC structures of different mineral mixtures.

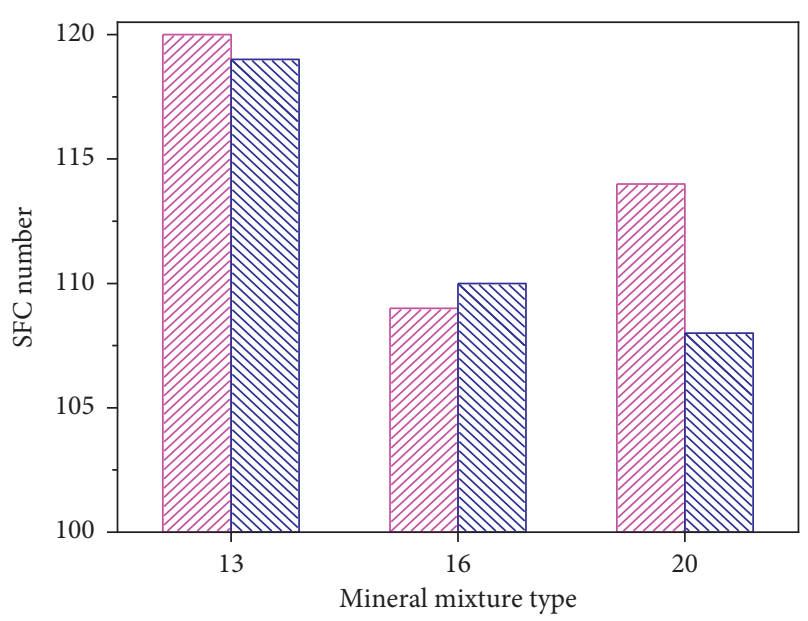

WIIA AC

VIIIA SMA

FIGURE 7: SFC number of different mineral mixtures.

Figure 11 shows the SFC cumulative proportions of dense-suspended and dense-skeleton mixtures. It can be found in Figure 11(a) that the SFC length cumulative proportions show a certain degree of discretization under different NMAS. The NMAS has a great influence on the SFC length cumulative proportions, and different NMAS can significantly change the stress transfer path for dense-suspended type mixtures. Nevertheless, for dense-skeleton type mixtures, the SFC length cumulative proportions have consistency. These findings reveal that, for dense-suspended type mixtures, the skeleton structure change is sensitive to NMAS. And different NMAS can significantly influence the stress transfer path for dense-suspended type mixtures. The dense-suspended type mixture with larger NMAS is beneficial to transfer external loading. We can also infer that the internal SFC length presents united distribution form for mixture with obvious skeleton structure which is beneficial to transfer external loading.

Figure 12 shows that the SMA mixtures have shorter average SFC length than that of AC mixtures. The NMAS has a great influence on the average SFC length of AC mixtures. However, the NMAS also has little influence on the average SFC length of SMA mixture. These findings are consistent with the results that are mentioned above. The short length SFC are conducive to transfer external loading. Therefore, dense-skeleton type mixture has better rutting resistance.

6.3. SFC Alignment Coefficient of Different Mineral Mixtures. The SFC alignment coefficient can reflect the stability of transfer external loading. In this study, the SFC alignment coefficient of mineral mixtures is also calculated by programming. And the SFC alignment coefficient distribution, minimum alignment coefficient of SFC, maximum alignment coefficient of SFC, and SFC alignment coefficient cumulative distribution of different mineral mixtures are shown in Figures 13 16, respectively.

Figure 13 shows that, on the whole, SFC alignment coefficient represents Gaussian distribution, and the peak value appears that the alignment coefficient is between 0.84 and 0.88 . Comparing the alignment coefficients of densesuspended dense-skeleton type mixtures under NMAS $13.2 \mathrm{~mm}$ shows that the small SFC alignment coefficient $(\leq 0.84)$ number proportions of $\mathrm{AC} 13$ and SMA13 are $24.17 \%$ and $20.17 \%$, as shown in Table 3 . These findings are consistent with the results shown in Figure 9. The long length SFC number proportion of $\mathrm{AC} 13$ is higher than that of SMA13. According to the meaning of alignment coefficient, the long length SFC are unstable. Therefore, the small SFC alignment coefficient $(\leq 0.84)$ number proportion of SMA13 is lower than that of AC13.

It can be also observed in Figures 13(b) and 13(c) that the small SFC alignment coefficient $(\leq 0.84)$ number proportions of AC16 and SMA16 are $23.85 \%$ and $20.91 \%$, respectively. That of AC20 and SMA20 is $36.84 \%$ and $18.52 \%$, respectively. These results further illustrate that the small SFC alignment coefficient number proportions of dense-suspended type mixtures are higher than that of dense-skeleton type mixtures. In particular, in the case of larger NMAS, the difference is more obvious. As discussed previously, the short length SFC number proportion of SMA is higher than that of AC. We infer that the larger alignment coefficient is, the more stable SFC is, and this is beneficial to bear and transfer loading. Therefore, compared with AC mixtures, SMA mixtures have better rutting resistance.

Figures 14 and 15 show the minimum and maximum alignment coefficients of different mixtures separately. It can 


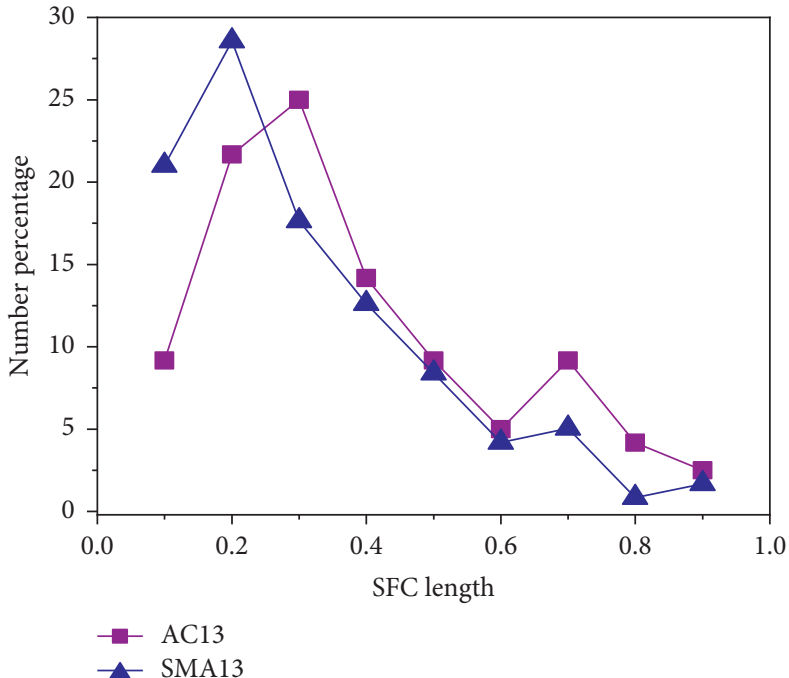

(a)

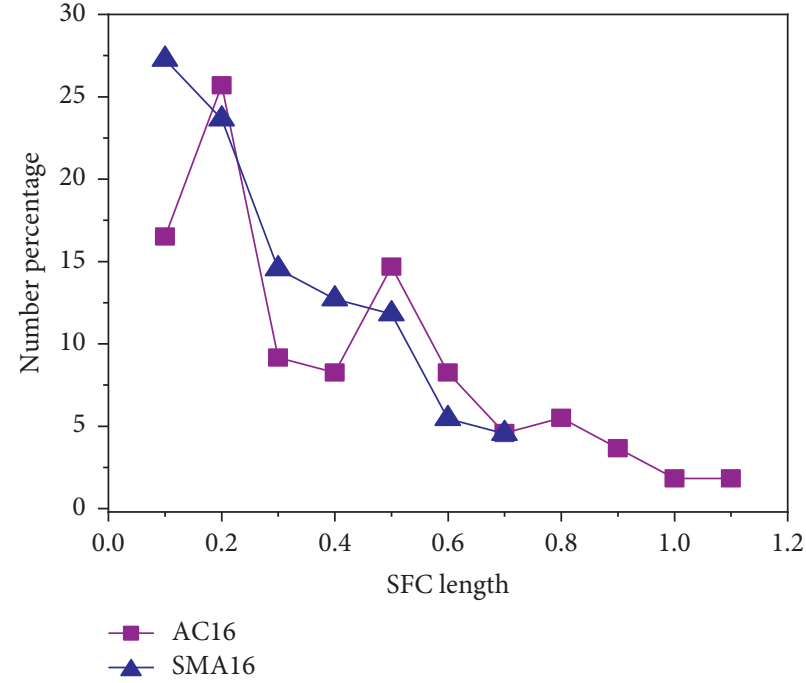

(b)

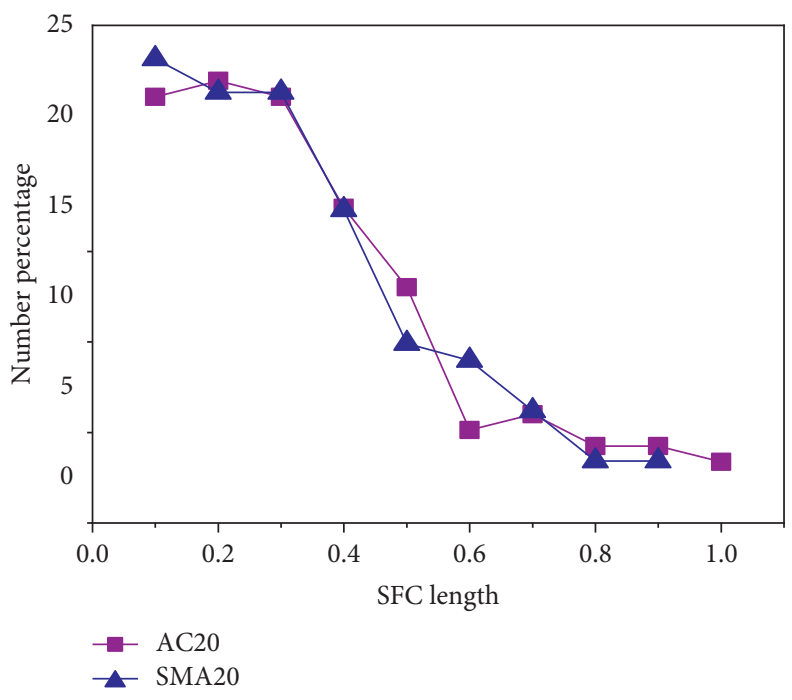

(c)

Figure 8: Dense-suspended type versus dense-skeleton type (SFC length distribution). (a) NMAS-13.2 mm, (b) NMAS-16 mm, and (c) NMAS-19 mm.

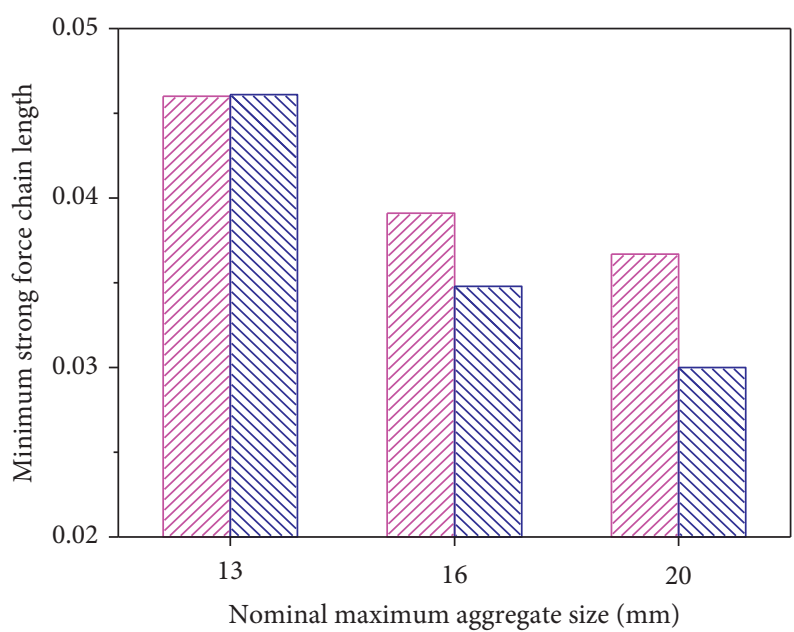

VIIA AC

VIII SMA

Figure 9: Minimum SFC length. 


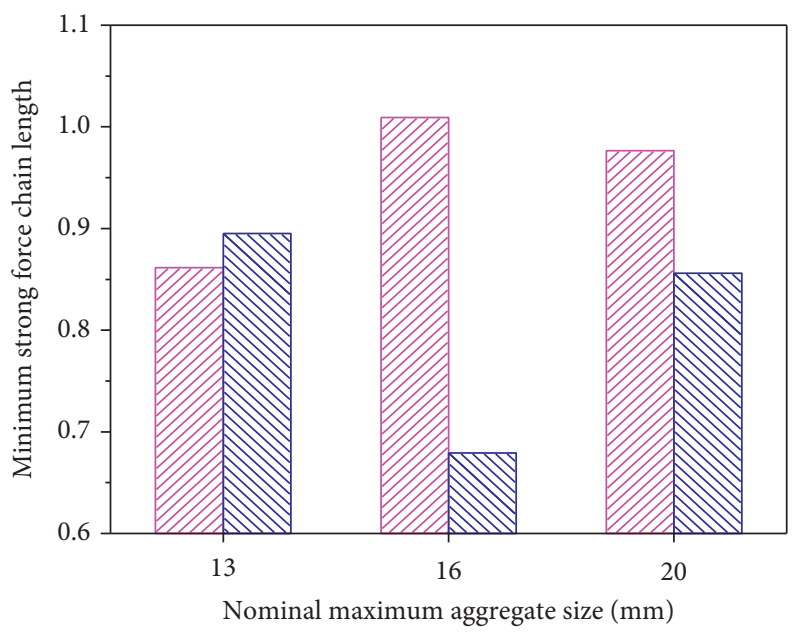

VIII AC

WIIA SMA

FIgURE 10: Maximum SFC length.

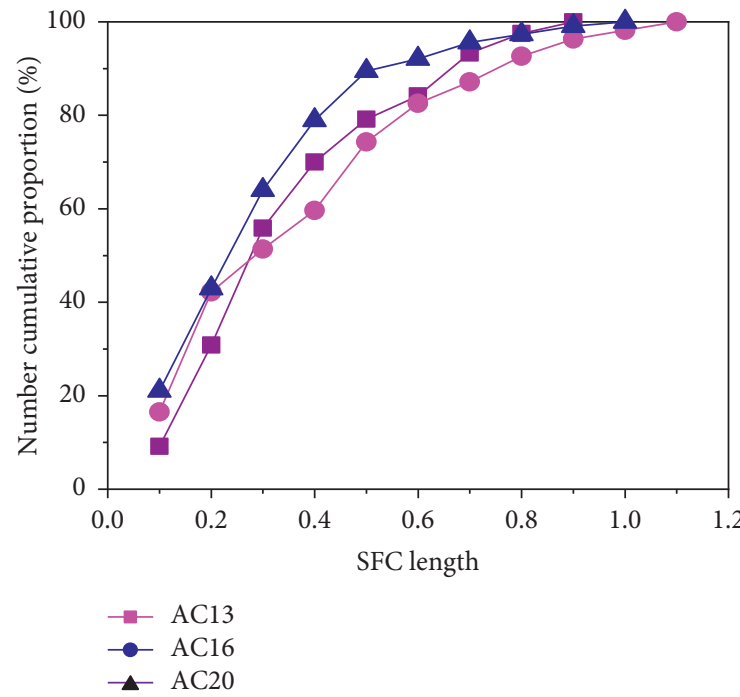

(a)

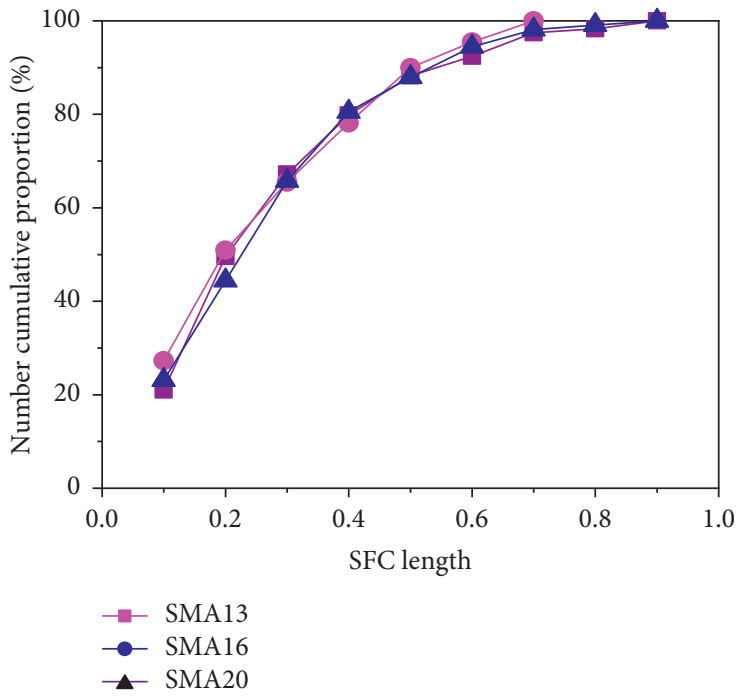

(b)

FiguRE 11: SFC number cumulative proportion. (a) Dense-suspended type and (b) dense-skeleton type.

TABLE 2: Short length SFC number proportions of different mineral mixtures.

\begin{tabular}{lc}
\hline Mineral mixture type & Short length SFC number proportion (\%) \\
\hline AC13 & 55.83 \\
AC16 & 51.38 \\
AC20 & 64.04 \\
SMA13 & 67.23 \\
SMA16 & 65.46 \\
SMA20 & 65.74 \\
\hline
\end{tabular}

be identified from Figure 14 that the minimum SFC alignment coefficients of AC mixtures are smaller than that of SMA mixtures. And Figure 15 shows that the maximum SFC alignment coefficients of AC and SMA mixtures are close to 1. According to the meaning of alignment coefficient, these SFC are close to straight lines which are beneficial to bear and transfer loading. Figure 15 also shows that the maximum SFC alignment coefficients of SMA16 and SMA20 mixtures are larger than the corresponding AC16 and AC20 mixtures, respectively. Although the maximum SFC alignment coefficient of SMA13 is smaller than the corresponding AC13, the large SFC alignment coefficient $(\geq 0.84)$ number proportion of SMA13 is higher than that of AC13, as shown in Figure 13.

Figure 16 shows the SFC alignment coefficient cumulative proportions of dense-suspended and dense-skeleton mixtures. It can be found in Figure 16(a) that the NMAS has a great influence on the SFC alignment coefficient cumulative proportions of dense-suspended type mixtures, and 


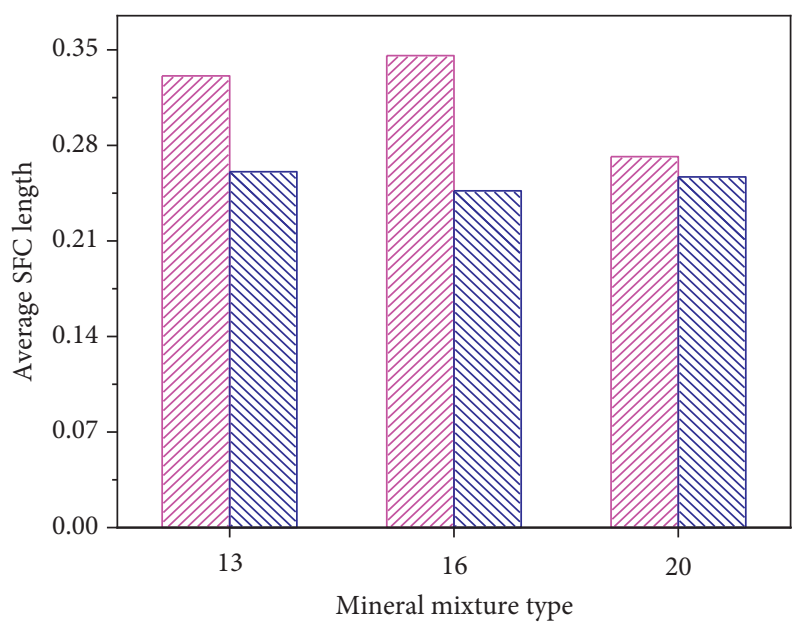

VIIA AC

VIII SMA

FIGURE 12: Average SFC length of different mineral mixtures.

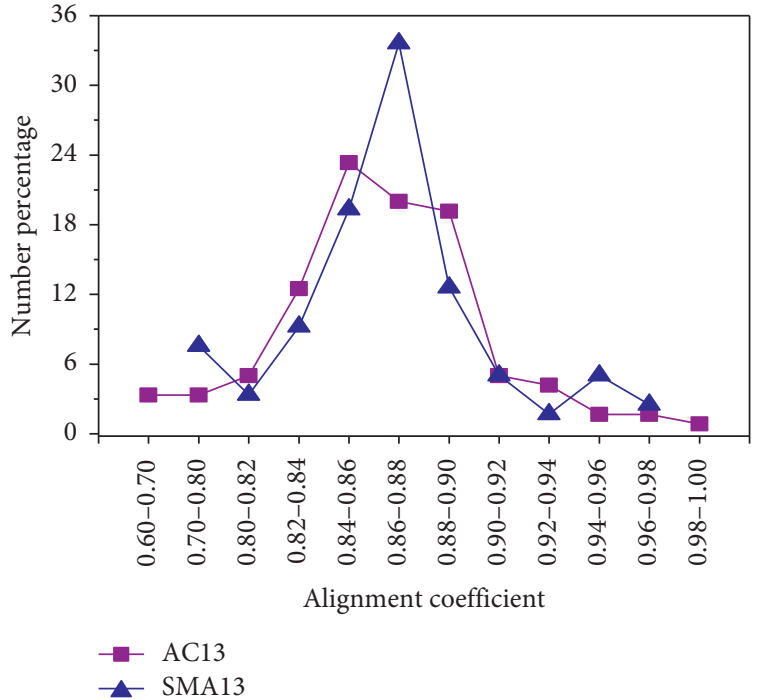

(a)

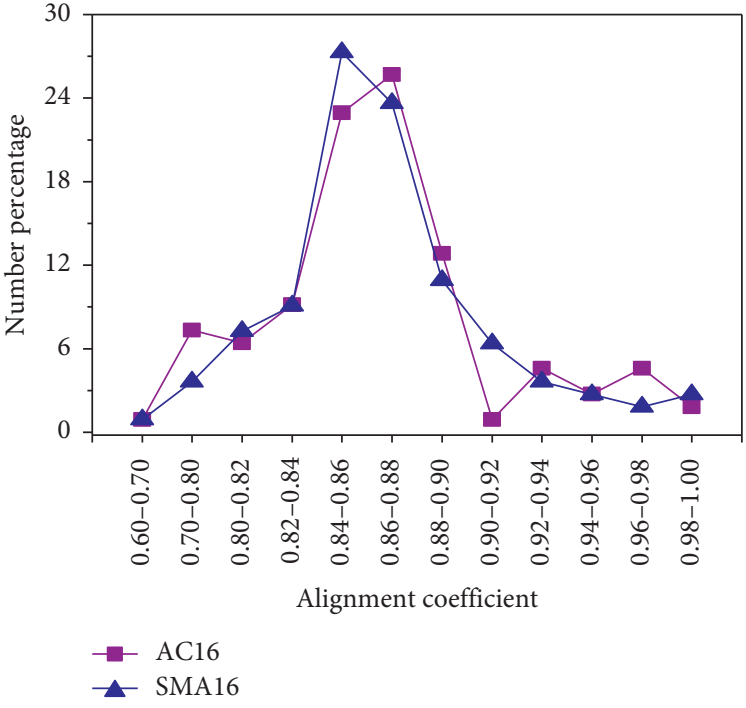

(b)

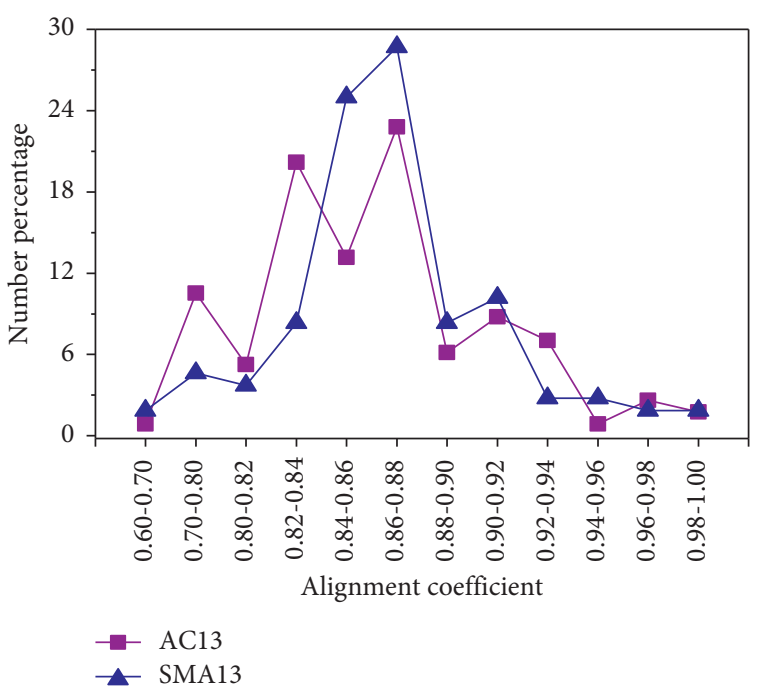

(c)

Figure 13: Dense-suspended type versus dense-skeleton type (SFC alignment coefficient distribution). (a) NMAS-13.2 mm, (b) NMAS$16 \mathrm{~mm}$, and (c) NMAS-19 mm. 


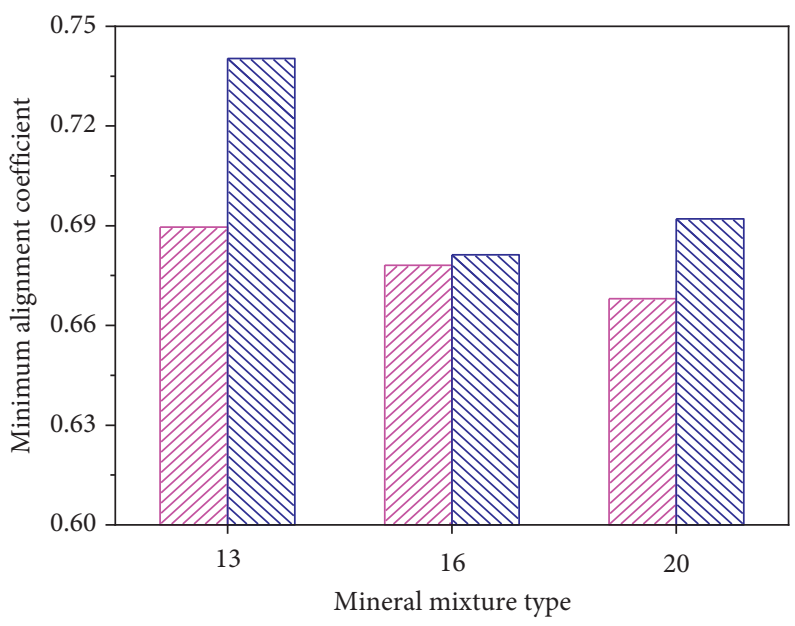

WIIA AC

VIIIA SMA

FIgUre 14: Minimum alignment coefficient.

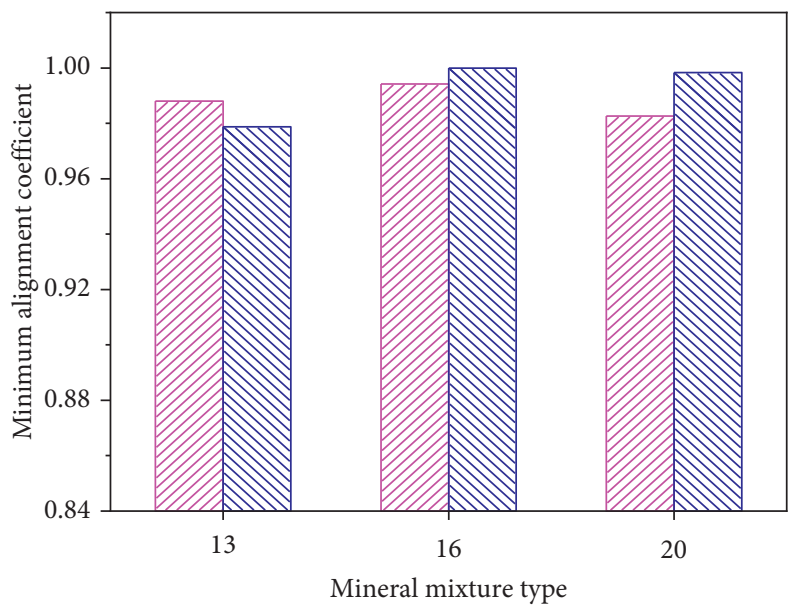

VIIIA AC

VIIIA SMA

FIGURE 15: Maximum alignment coefficient.

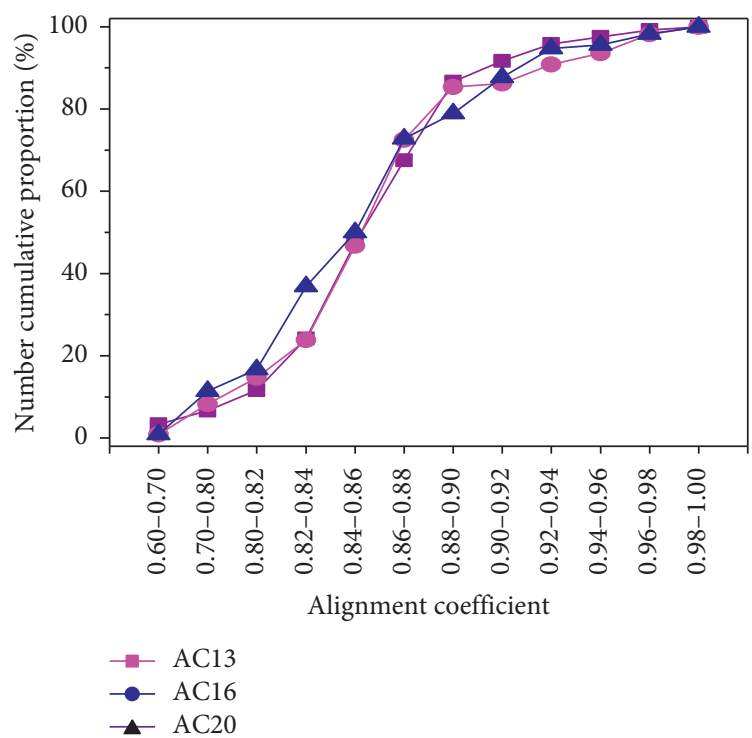

(a)

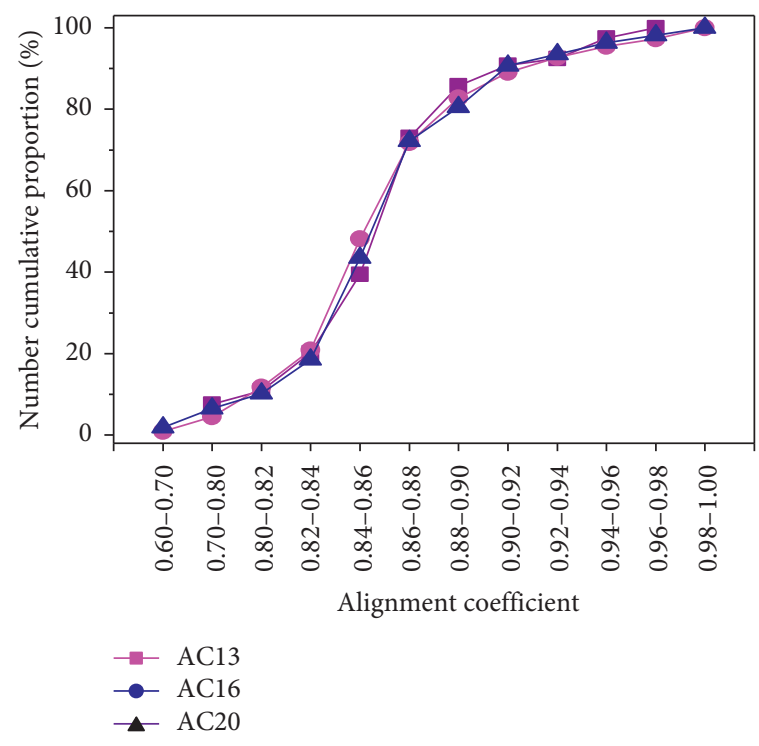

(b)

FIGURE 16: SFC alignment coefficient cumulative proportion. (a) Dense-suspended type and (b) dense-skeleton type. 
TABLE 3: Small SFC alignment coefficient number proportion of different mineral mixtures.

\begin{tabular}{lc}
\hline Mineral mixture type & $\begin{array}{c}\text { Small SFC alignment coefficient number } \\
\text { proportion (\%) }\end{array}$ \\
\hline AC13 & 24.17 \\
AC16 & 23.85 \\
AC20 & 36.84 \\
SMA13 & 20.17 \\
SMA16 & 20.91 \\
SMA20 & 18.52 \\
\hline
\end{tabular}

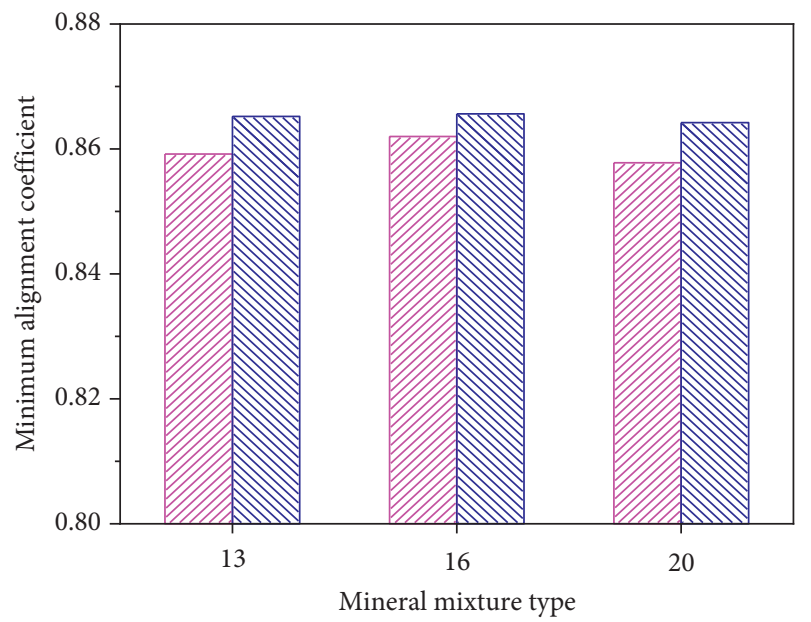

VII AC

VIIA SMA

FIgURe 17: Average SFC alignment coefficient of different mineral mixtures.

NMAS can significantly change the stress transfer path for dense-suspended type mixtures. Nevertheless, the SFC alignment coefficient cumulative proportions have consistency for dense-skeleton type mixtures. As mentioned before, the internal SFC presents united distribution form for mixture with obvious skeleton structure which is beneficial to transfer loading.

It can be seen from Figure 17 that the SMA mixtures have larger average SFC alignment coefficient than that of $\mathrm{AC}$ mixtures. The NMAS has a great influence on the average SFC alignment coefficient of AC mixtures, and the NMAS also has little influence on the average SFC alignment coefficient of SMA mixtures. These findings are consistent with the results that are mentioned above. The SFC that are close to straight line are conducive to transfer loading. Therefore, dense-skeleton type mixture has better rutting resistance.

6.4. SFC Bearing Capacity. The SFC bearing ratio of different mineral mixtures is calculated by (8), as shown in Figure 18.

Figure 18 shows that SFC bearing ratios of AC and SMA mixtures are more than $80 \%$ which indicate that the SFC bears the main external loading for mixtures and the validity of the SFC decision criteria. It can be also illustrated by Figure 18 that SMA mixtures have a stronger bearing

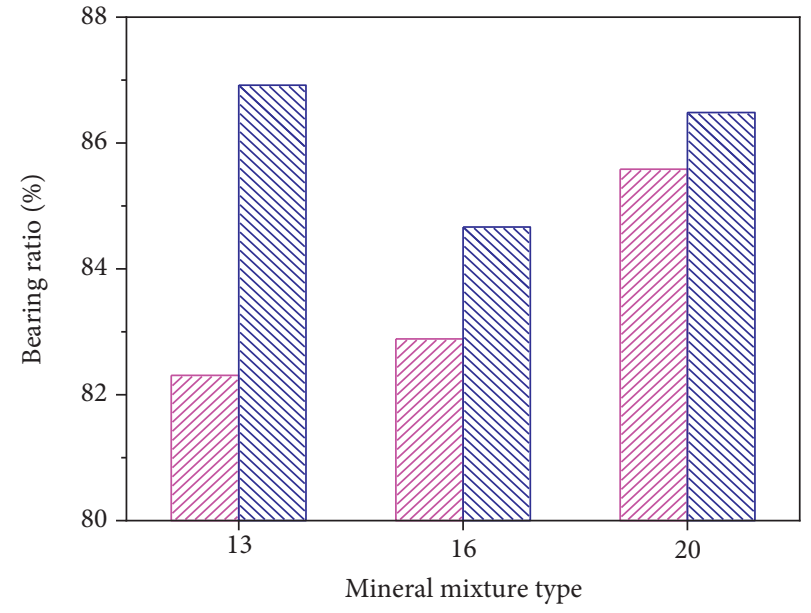

VII] AC

VIIA SMA

FIGURE 18: SFC bearing ratio of different mineral mixtures.

capacity than that of AC mixtures under the same NMAS. And the bearing capacity difference between AC and SMA mixtures decreases with an increase in NMAS which indicates that, for mixtures that skeleton structure is not evident, the increase of coarse aggregate particle size is beneficial to improve the SFC bearing capacity of mixtures. However, for mixtures that skeleton structure is evident, the increase of coarse aggregate particle size does not increase the SFC bearing capacity of mixtures significantly.

\section{Conclusions}

Based on the above simulation results and analysis, the following conclusions can be drawn:

(1) The short length SFC number proportions of denseskeleton type mixtures are higher than that of densesuspended type mixtures under the same NMAS. The short SFC are more stable than long SFC and are beneficial to bear and transfer loading.

(2) The NMAS can significantly change the stress transfer path for dense-suspended type mixtures. Nevertheless, the SFC length cumulative proportions have consistency for dense-skeleton type mixtures.

(3) The small SFC alignment coefficient number proportions of dense-suspended type mixtures are higher than that of dense-skeleton type mixtures. In particular, in the case of larger NMAS, the difference is more obvious.

(4) The SFC that is close to straight lines is conducive to transfer loading. And the SFC bears the main loading for mixtures. Dense-skeleton type mixtures have a stronger bearing capacity than that of dense-suspended type mixtures under the same NMAS.

\section{Data Availability}

The data used to support the findings of this study are included within the article. 


\section{Conflicts of Interest}

The authors declare no conflicts of interest.

\section{Authors' Contributions}

All authors approved the manuscript.

\section{Acknowledgments}

This study was supported by National Key Research and Development Program of China (2018YF-B1600200) and Research on Key Technology of Hot In-Place Recycling for Rubber Asphalt Pavements (BY2015070-04). The authors gratefully acknowledge their financial support.

\section{References}

[1] J. Li, M. Shang, G. Liu et al., "Two-step improvements of volumetric design method based on multi-point supported skeleton for asphalt mixtures," Construction and Building Materials, vol. 217, pp. 456-472, 2019.

[2] X. Ding, T. Ma, and W. Gao, "Morphological characterization and mechanical analysis for coarse aggregate skeleton of asphalt mixture based on discrete-element modeling," Construction and Building Materials, vol. 154, pp. 1048-1061, 2017.

[3] A. R. Coenen, M. E. Kutay, N. R. Sefidmazgi, and H. U. Bahia, "Aggregate structure characterisation of asphalt mixtures using two-dimensional image analysis," Road Materials and Pavement Design, vol. 13, no. 3, pp. 433-454, 2012.

[4] X. Cai and D. Wang, "Evaluation of rutting performance of asphalt mixture based on the granular media theory and aggregate contact characteristics," Road Materials and Pavement Design, vol. 14, no. 2, pp. 325-340, 2013.

[5] N. Roohi Sefidmazgi and H. U. Bahia, "Effect of compaction conditions on aggregate packing using 2-dimensional image analysis and the relation to performance of HMA," Materials and Structures, vol. 47, no. 8, pp. 1313-1324, 2014.

[6] N. R. Sefidmazgi, L. Tashman, and H. Bahia, "Internal structure characterization of asphalt mixtures for rutting performance using imaging analysis," Road Materials and Pavement Design, vol. 13, no. 1, pp. 21-37, 2012.

[7] N. R. Sefidmazgi, P. Teymourpour, and H. U. Bahia, "Effect of particle mobility on aggregate structure formation in asphalt mixtures," Road Materials and Pavement Design, vol. 14, no. 2, pp. 16-34, 2013.

[8] L. W. Shi, D. Y. Wang, X. Cai et al., "Distribution characteristics of coarse aggregate contacts based on digital image processing technique," China Journal of Highway and Transport, vol. 27, no. 8, pp. 23-31, 2014.

[9] L. W. Shi and D. Y. Wang, "Evaluation indexes of asphalt mixture main skeleton based on digital image processing," China Journal of Highway and Transport, vol. 30, no. 5, pp. 52-58, 2017.

[10] H. Ying, J. Zhou, Q. Wu et al., "Variation of the contact form coarse aggregate particles in skeleton type Asphalt mixture," Journal of Building Materials, vol. 19, no. 02, pp. 292-298, 2016.

[11] J. Jiang, F. Ni, L. Gao, and L. Yao, "Effect of the contact structure characteristics on rutting performance in asphalt mixtures using 2D imaging analysis," Construction and Building Materials, vol. 136, pp. 426-435, 2017.
[12] J. Jiang, F. Ni, Q. Dong, L. Yao, and X. Ma, "Investigation of the internal structure change of two-layer asphalt mixtures during the wheel tracking test based on 2D image analysis," Construction and Building Materials, vol. 209, pp. 66-76, 2019.

[13] J. Chen, H. Li, L. Wang, J. Wu, and X. Huang, "Micromechanical characteristics of aggregate particles in asphalt mixtures," Construction and Building Materials, vol. 91, pp. 80-85, 2015.

[14] T. Ma, D. Zhang, Y. Zhang, and J. Hong, "Micromechanical response of aggregate skeleton within asphalt mixture based on virtual simulation of wheel tracking test," Construction and Building Materials, vol. 111, pp. 153-163, 2016.

[15] P. Li, Z. Ding, and W. Rao, "Evaluation of deformation properties of asphalt mixture using aggregate slip test," International Journal of Pavement Engineering, vol. 17, no. 6, pp. 542-549, 2016.

[16] X. Wang, X. Gu, J. Jiang, and H. Deng, "Experimental analysis of skeleton strength of porous asphalt mixtures," Construction and Building Materials, vol. 171, pp. 13-21, 2018.

[17] Y. Zhang, X. Luo, I. Onifade, X. Huang, R. L. Lytton, and B. Birgisson, "Mechanical evaluation of aggregate gradation to characterize load carrying capacity and rutting resistance of asphalt mixtures," Construction and Building Materials, vol. 205, pp. 499-510, 2019.

[18] Q. Sun, F. Jin, J. Liu, and G. Zhang, "Understanding force chains in dense granular materials," International Journal of Modern Physics B, vol. 24, no. 29, pp. 5743-5759, 2010.

[19] Y. Chen and Z. Li, "Meso-structure of crumb rubber asphalt mixture based on discrete element method," Journal of Harbin Institute of Technology, vol. 45, no. 4, pp. 116-121, 2013.

[20] L. Shi, D. Wang, C. Xu et al., "Investigation into meso performance of asphalt mixture skeleton based on discrete element method," Journal of South China Univeristy of Technology (Natrue Science Edition), vol. 43, no. 10, 2015.

[21] M. Chang, P. Huang, J. Pei, J. Zhang, and B. Zheng, "Quantitative analysis on force chain of asphalt mixture under haversine loading," Advances in Materials Science and Engineering, vol. 2017, pp. 1-7, 2017.

[22] J. Pei, "Progress of highway engineering and generation upgrading of highway transportation system," China Journal of Highway and Transport, vol. 31, no. 11, pp. 1-10, 2018.

[23] Ministry-of-Transport-of-the-People's-Republic-of-China, Technical Specification for Construction of Highway Asphalt Pavement JTG F40-2004, China Communication Press, Beijing, China, 2004.

[24] Y. Liu, Q. Dai, and Z. You, "Viscoelastic model for discrete element simulation of asphalt mixtures," Journal of Engineering Mechanics, vol. 135, no. 4, pp. 324-333, 2009.

[25] Z. You, S. Adhikari, and Q. Dai, "Three-dimensional discrete element models for asphalt mixtures," Journal of Engineering Mechanics, vol. 134, no. 12, pp. 1053-1063, 2008.

[26] X. Ding, T. Ma, and X. Huang, "Discrete-element contourfilling modeling method for micromechanical and macromechanical analysis of aggregate skeleton of asphalt mixture," Journal of Transportation Engineering, Part B: Pavements, vol. 145, no. 1, Article ID 04018056, 2019.

[27] S. Shen and H. Yu, "Characterize packing of aggregate particles for paving materials: particle size impact," Construction and Building Materials, vol. 25, no. 3, pp. 1362-1368, 2011.

[28] M. E. Cates, J. P. Wittmer, J.-P. Bouchaud, and P. Claudin, "Jamming, force chains, and fragile matter," Physical Review Letters, vol. 81, no. 9, pp. 1841-1844, 1998. 
[29] D. Howell, R. P. Behringer, and C. Veje, "Stress fluctuations in a 2D granular Couette experiment: a continuous transition," Physical Review Letters, vol. 82, no. 26, pp. 5241-5244, 1999.

[30] J. F. Peters, M. Muthuswamy, J. Wibowo et al., "Characterization of force chains in granular material," Physical Review E, vol. 72, no. 4, 2005.

[31] Q. C. Sun and G. Q. Wang, "Force distribution in static granular matter in two dimensions," Acta Physica Sinica, vol. 57, no. 8, pp. 4667-4674, 2008. 